\title{
Duktales Carcinoma in situ (DCIS)
}

\section{Ductal Carcinoma in situ (DCIS)}

\author{
Gesprächsleitung: Raimund Jakesz (Wien) \\ Teilnehmer: $\quad$ Christoph Jakisch, Offenbach Walter Jonat/Christoph Mundhenke, Kiel \\ Christian Marth, Halle (Saale) Michael Stierer, Wien \\ Augustinus H. Tulusan, Bayreuth
}

\section{Frage 1: Welchen Stellenwert hat die Radiatio bei brusterhaltender Operation wegen DCIS?}

Jonat/Mundhenke: Wir empfehlen die postoperative Radiatio nach Brusterhaltung. Bei Befunden mit niedrigem Risiko verzichten wir gegebenenfalls auf eine Radiatio (z.B. bei «Nonhigh grade»-DCIS ohne Nekrosen und tumorfreien Resektionsrändern über $10 \mathrm{~mm}$ ). Die Rationale für die postoperative Bestrahlung der Restbrust nach DCIS ergibt sich aus den Ergebnissen der NSABP-17-Studie, die eine deutliche Risikoreduktion hinsichtlich des Auftretens von nichtinvasiven und invasiven Lokalrezidiven nach Radiatio zeigt.

Marth: Da die Bedeutung des DCIS erst in den 1980er Jahren langsam klar wurde, ist für dessen Behandlung die Studienlandschaft noch relativ karg; insbesondere fehlen Langzeiterfahrungen. Die Strahlentherapie als obligate Komponente der brusterhaltenden Therapie wurde beim DCIS sehr kontrovers diskutiert. Die Entfernung des DCIS im Gesunden kombiniert mit Strahlentherapie erlaubt eine ausreichende lokale Kontrolle. Allerdings stellt die Strahlentherapie in vielen Fällen eine «Über-Therapierung» dar, da die Mehrheit der Patientinnen mit DCIS weder ein Rezidiv noch einen invasiven Tumor entwickeln, sofern sie chirurgisch optimal behandelt wurden. Die Ergebnisse der NSABP-B17-Studie wurden dahingehend interpretiert, dass alle Patientinnen mit DCIS und brusterhaltender Therapie nachbestrahlt werden sollen. Allerdings wurde diese Studie zu einer Zeit begonnen, in der man über die Bedeutung des DCIS noch wenig wusste und dadurch viele Patientinnen nicht adäquat chirurgisch behandelte. Dies erklärt die inakzeptabel hohe Rezidivrate in der Kontrollgruppe von $32 \%$, die durch die Strahlentherapie in etwa halbiert werden konnte. Vergleichbare spätere Untersuchungen wie die EORTC-10853 oder die in England, Australien und Neuseeland durchgeführte DCIS-Studie (UK/ANZ-Studie) konnten ebenfalls einen Effekt der Strahlentherapie nachweisen (Rezidivreduktion um etwa 50\%). Obwohl alle Untergruppen von der Strahlentherapie profitierten, sind vor allem jüngere Patientinnen aufgrund des höheren Lokalrezidivrisikos jene mit dem größten Benefit. In diesen Studien war die Lokalrezidivrate in der Kontrollgruppe deutlich geringer. Diese Studien belegen einhellig, dass mit einer alleinigen Strahlentherapie Rezidive beim DCIS nur insuffizient verhindert werden können und dass eine Resektion im Gesunden als zentrale Anforderung an die primäre Therapie gesehen werden muss. Das Ziel ist die Identifizierung derjenigen Patientinnen, die am meisten von einer Strahlentherapie profitieren. Wir verwenden hierzu den VanNuys-Prognose-Index. Bei 6 oder weniger VanNuys-Punkten kann von einer 99\%igen 10-Jahres-Rezidiv-Freiheit ausgegangen werden. In dieser Untergruppe von DCIS-Patientinnen sollte die Nebenwirkung der Strahlentherapie nicht akzeptiert werden.

Stierer: Die adjuvante postoperative Radiatio stellt derzeit einen integrierenden Bestandteil in der Therapie des DCIS dar. In allen prospektiven und fast allen retrospektiven Untersuchungen konnte durch diese Maßnahme eine bis zu $60 \%$ ige, signifikante Reduktion der Lokalrezidivrate ohne Einfluss auf die Gesamtsterblichkeit erzielt werden. Dieser Effekt kommt wahrscheinlich dadurch zustande, dass alle Studien im Hinblick auf die Evaluierung dieser Fragestellung zu wenige Patientinnen aufgewiesen haben. Letztlich konnte der negative Einfluss eines Lokalrezidivs auf die Mortalität beim invasiven Karzinom auch nur im Rahmen einer MetaAnalyse zahlreicher Studien nachgewiesen werden. Jedenfalls ist aus kleinen Serien eine Mortalitätsrate von zirka 14\% durch das Auftreten eines invasiven Rezidivs nach operiertem DCIS bekannt. Aber auch die Verhinderung eines intra-

\begin{tabular}{ll}
\hline KARGER & @ 2006 S. Karger GmbH, Freiburg \\
Fax +49 761 4520714 & Accessible online at: \\
$\begin{array}{l}\text { E-mail Information@Karger.de } \\
\text { www.karger.com }\end{array}$ & www.karger.com/brc \\
&
\end{tabular}

Univ. Prof. Dr. Dr. h.c. Raimund Jakesz

Klinische Abteilung für Allgemeinchirurgie

Medizinische Universität Wien

Währinger Gürtel 18-20, 1090 Wien, Österreich

Tel. +43 1 40400-6916, Fax -6918

E-mail raimund.jakesz@meduniwien.ac.at 
duktalen Rezidivs ist zur Vermeidung einer Zweitoperation mit fraglicher Brusterhaltung sowie auch aus psychologischen Gründen sinnvoll. Allerdings stellt sich bei insgesamt ungünstiger Risk-Benefit-Ratio (Bestrahlung von 36 Patientinnen zur Vermeidung eines Rezidivs notwendig) die Frage, ob nicht bei speziellen Subgruppen auf die Radiotherapie verzichtet werden kann. Neben lokalen Nebenwirkungen sowie einem erschwerten radiologischen Follow-up stellt sich hier auch die Kostenfrage.

Die retrospektiven Daten von Silverstein MJ, et al. [The influence of margin width on local control of ductal carcinoma in situ of the breast. N Eng J Med 1999;340:1455-1461] zeigen, dass bei Erzielung von mehr als $10 \mathrm{~mm}$ freien Schnitträndern auch ohne Nachbestrahlung nach einem mittleren Follow-up von 8 Jahren eine excellente Rezidivrate von zirka 6\% erzielbar ist. Das Erreichen solcher Resultate setzt allerdings ein sehr arbeitsaufwändiges histologisches «work-up» mit obligater Korrelierung zur mammographischen Ausbreitung voraus, welches wahrscheinlich derzeit flächendeckend noch nicht zur Verfügung steht, wohl aber ein gutes Argument für eine auf spezialisierte Zentren beschränkte Behandlung des DCIS darstellt. Ebenso ist man unter Wahrung dieser Schnittrandweite bei Vorliegen einer durchschnittlich großen Brust schon bei mittelgroßen Läsionen mit einer beträchtlichen kosmetischen Problematik konfrontiert.

Im Einzelfall könnte bei einer über 40-jährigen Patientin mit Vorliegen eines DCIS G1, G2, kleiner als $15 \mathrm{~mm}$ und einer Schnittrandweite über $10 \mathrm{~mm}$ im Sinne eines «informed consent» auf die Strahlentherapie verzichtet werden.

Es ist zu hoffen, dass laufende und bereits abgeschlossene Trials (ECOG E 5194, RTOG 98-04, BASO) die Frage der Notwendigkeit einer obligaten Radiotherapie bzw. eines Boosts bei einer G3-Läsion beantworten helfen.

Tulusan: Je ungenauer die pathohistologische Aufarbeitung (Unklarheit über Ausdehnung/Größe des DCIS, «Margin-Status», unentdeckte Invasionsherde usw.) ist, desto größer sind die Vorteile der zusätzlichen Radiatio. Je genauer die pathohistologische Aufarbeitung, desto differenzierter ist die Therapiemöglichkeit in Hinblick auf die Radiatio. Trotz Radiatio haben zahlreiche Studien gezeigt, dass die Lokalrezidivraten noch deutlich höher liegen, wenn die Aussage über den Resektionsrand ungünstig ist! Demnach ist die Strahlentherapie effektiv, stellt aber keinen Ersatz für die kurative Operation der «freien» Resektionsränder dar. Dies relativiert die Bedeutung der zusätzlichen Radiatio bei DCIS!

Wichtigste Voraussetzung ist die Implementierung der Therapieprinzipien:

- sektoriale Segmentresektion zur vollständigen Entfernung des DCIS,

- «Margins» zumindest $1 \mathrm{~cm}$,

- vollständige Aufarbeitung des Resektats (nach Konsensus DCIS 2001),

- genauer Malignitätsgrad des DCIS (Lagios).
Die Implementierung der Therapieprinzipien erfordert eine sehr enge interdisziplinäre Zusammenarbeit der beteiligten Fachdisziplinen (Radiologie, Chirurgie, Radiotherapie, Pathologie).

Alle Studien (auch randomisierte) mit Abweichungen von den Therapieprinzipien haben eine sehr hohe Lokalrezidivrate nach brusterhaltender Therapie ohne Bestrahlung (siehe oben) mit Lokalrezidiven in bis zu 39\%(!) der Fälle, während die prospektiven Studien mit genauer Applikation der Therapieprinzipien eine niedrige Lokalrezidivrate haben (0-7\%), auch ohne Strahlentherapie.

Empfehlungen:

- Bei G1, G2, G3 und «Margins» > 1 cm keine Radiatio,

- Bei «Margins» $<1 \mathrm{~cm}$ bis $5 \mathrm{~mm}$, wenn möglich, Nachresektion,

- Bei «Margins» $<5 \mathrm{~mm}$ bis $1 \mathrm{~mm}$ Nachresektion oder Radiatio,

- Bei «Margins» non in sano (transection) oder $<1 \mathrm{~mm}$ Nachresektion, auch wenn Radiatio oder Ablatio.

Alternativ (Bei Anwendung des VNPI):

- VNPI 3-4 keine Radiatio,

- VNPI 5-7 Nachresektion oder Radiatio,

- VNPI 8-9 Nachresektion, Radiatio oder Ablatio.

Bei Anwendung des neuen VNPI mit Berücksichtigung des Alters:

- VNPI 4-6 keine Radiatio,

- VNPI 7-9 Nachresektion oder Radiatio,

- VNPI 10-12 Nachresektion, Radiatio oder Ablatio.

Eigene Erfahrung mit 191 DCIS (Bayreuth, Erlangen) zeigen

folgende Verteilung: 32\% (VNPI 3-4), 38\% (VNPI 5-7), 30\% (VNPI 8-9).

\section{Frage 2: Wie gesichert ist die Antihormontherapie? Ist die Bestimmung des Rezeptors beim DCIS obligat?}

Jonat/Mundhenke: In Kiel wird bei jedem DCIS routinemäßig der Hormonrezeptorstatus bestimmt. Nach Möglichkeit werden Patientinnen mit einem positiven Rezeptorstatus im Rahmen der IBIS-II-Studie behandelt. In dieser Studie wird quasi adjuvant die Applikation von Tamoxifen gegen den Aromatase-Inhibitor Anastrozol verglichen. In der abgeschlossenen NSABP-B24-Studie hatte die Einnahme von Tamoxifen nach DCIS bei positivem Hormonrezeptorstatus zu einer Verringerung des Lokalrezidivrisikos geführt. Unter Tamoxifen war jedoch die Rate unerwünschter Nebenwirkungen, wie z.B. das Auftreten von Thrombosen und Endometriumkarzinomen (zirka 3,5-fach) erhöht. Da die Nutzen-Risiko-Analyse bislang keine eindeutigen Vorteile erbracht hat, verordnen wir routinemäßig keine endokrine Therapie nach DCIS. Falls eine Studienteilnahme nicht möglich ist, entscheiden wir jedoch in Einzelfällen in Abhängigkeit vom Risikoprofil gemeinsam mit der Patientin über mögliche medikamentöse Therapieoptionen. 
Marth: In Analogie zum invasiven Karzinom wird beim DCIS eine Rezeptorbestimmung empfohlen. Etwa die Hälfte der Tumoren exprimiert im intraduktalen Anteil den Östrogen- und/oder den Progesteronrezeptor. Die NSABPB24-Studie konnte für diese Patientinnen eine deutliche Reduktion des Rezidivrisikos, (RR 0,41, absolute Differenz etwa $4 \%$ ) durch eineTamoxifentherapie belegen. Hormonrezeptornegative Patientinnen profitierten hingegen nicht von der adjuvanten Antihormontherapie. Im Gegensatz zu dieser Studie hat die UK/ANZ-Studie (insgesamt 1700 Patientinnen) keinen wesentlichen Vorteil einer adjuvanten Tamoxifen-Therapie in der rezeptorpositiven Gruppe nachweisen können. Lediglich in der Untergruppe «brusterhaltende Operation, ohne Strahlentherapie» wurde ein geringer Nutzen der endokrinen Therapie beobachtet. Wenn man gleichzeitig die negativen Effekte der Antiöstrogen-Langzeitbehandlung in Betracht zieht (Endometriumhyperplasie und Endometriumkarzinom, Uterussarkome, thromboembolische Komplikationen usw.), kann bei einem bisher nicht nachgewiesenen Überlebensvorteil durch die adjuvante Tamoxifen-Therapie diese nicht uneingeschränkt empfohlen werden. Weitere Studien, wie z.B. IBIS II, müssen klären, ob und welche Form die adjuvante Hormontherapie beim DCIS $\mathrm{zu}$ empfehlen ist.

Stierer: Die Bedeutung einer adjuvanten Hormontherapie mit Tamoxifen beim operierten DCIS zur Senkung der Lokalrezidivrate und zur Reduktion der Frequenz eines kontralateralen Karzinoms wurde in zwei prospektiven Studien geprüft. In der NSABP-B-24-Studie (Follow-up-Zeit 109 Monate) konnte eine signifikante Reduktion der Lokalrezidivrate durch die Gabe von Tamoxifen nach Strahlentherapie vor allem bei unter 50-jährigen Patientinnen gezeigt werden; insgesamt wurde das Risiko des Auftretens von Brustkrebs (homolateral, kontralateral, invasiv oder präinvasiv) jedoch signifikant von 16,9 auf $10 \%$ reduziert [Fisher B, et al: Prevention of invasive breast cancer in women with ductal carcinoma in situ: an update of the national surgical adjuvant breast and bowel project experience. Semin Oncol 2001;28:400-418]. Erwartungsgemäß ergab eine retrospektive Rezeptoranalyse, dass die Wirksamkeit des Antiöstrogens auf hormonrezeptorpositive Fälle beschränkt war.

In der UK/ANZ-Studie mit einem komplexem $2 \times 2$-Design $(\mathrm{OP} \pm$ Radiotherapie \pm Tamoxifen $)$ und mit kürzerer medianer Beobachtungszeit von 52,5 Monaten konnte durch das Antiöstrogen kein signifikanter Effekt erzielt werden. Die adjuvante Gabe von Tamoxifen bleibt daher außerhalb von Studien nur eine Therapieoption, vor allem für Patientinnen unter 50 Jahren, mit hormonrezeptorpositivem DCIS, weil hier ein günstiges Nebenwirkungsrisikoprofil vorliegt. Ebenso kann bei Grad-3-Läsionen mit knappem Schnittrand und Ablehnung einer Reexzision bei hormonrezeptorpositivem DCIS, insbesondere bei Zustand nach Hysterektomie, die Indikation diskutiert werden. Dies gilt auch allgemein für jünge- re postmenopausale Patientinnen mit hormonrezeptorpositiven Grad-III-Läsionen ohne Komorbidität.

Die Rolle der Aromatasehemmer wird derzeit in zwei Studien (NSABP B-35, IBIS II) untersucht.

In einer Pilotstudie [Hwang ES, Esserman L: Neoadjuvant hormonal therapy for ductal carcinoma in situ: trial design and preliminary results. Ann Surg Oncol 2004;11(suppl 1): 37S-43S] konnte auch gezeigt werden, dass die präoperative Gabe von Tamoxifen und Letrozol zu einer fast 50\%igen Volumenreduktion und zu einem signifikantem Abfall des Proliferationsmarkers KI 67 führt. Diese präliminaren Ergebnisse müssten in einer randomisierten Studie weiter geprüft werden.

Tulusan: Tamoxifen wurde und wird als Therapie oder zur Prävention beim Mammakarzinom verwendet. Positive Östrogenrezeptoren sind bisher vorwiegend bei höher differenziertem DCIS vom Non-comedo-Typ nachweisbar. Beim DCIS sind bis auf die Studienergebnisse von Fisher (NSABP B-24) und dem UK/ANZ-Trial keine weiteren entscheidenden Untersuchungsergebnisse zu Tamoxifen bekannt. Die bisherigen Daten zeigen nur geringe absolute Vorteile der Verwendung von Tamoxifen für die Verhinderung des Lokalrezidivs und eher eine Prophylaxe für die Entstehung von neuen Erkrankungen in der kontralateralen Brust. Dieser mögliche, geringe Vorteil aus der B24-Studie war in der UK/ANZ-Studie allerdings nicht reproduzierbar. So ist die Verwendung von Tamoxifen beim Östrogenrezeptor-positiven DCIS zu empfehlen, wenn die Patientin keine weiteren Risikofaktoren für Tamoxifen (z.B. Thromboserisiko) aufweist und mit der Therapie keine wesentlichen Nebenwirkungen einhergehen. $\mathrm{Ob}$ Aromataseinhibitoren ein besseres Ergebnis zeigen können, ist noch Gegenstand von laufenden Studien (z.B. IBIS II).

Jakisch: Im Rahmen der zunehmenden Frequenz des populationsbezogenen Mamma-Screenings in Deutschland werden wir eine weitere Steigerung der Detektion von DCIS in den entsprechenden Altersgruppen antreffen. Neben einer sicheren operativen Therapie erscheint mir der Einsatz von Tamoxifen zur Absenkung der des intramammären Rezidivs und der Prävention des invasiven Mammakarzinoms bei positivem Östrogenrezeptorstatus unverzichtbar. Das gilt darüber hinaus natürlich auch für die Prävention des kontralateralen Karzinoms. Der Einsatz von Aromatasehemmern bei DCIS sollte außerhalb der IBIS-II-Studie unterbleiben.

\section{Frage 3: DCIS und Brusterhaltung: Welche Bedeutung hat die Mammographie in der Nachsorge?}

Jonat/Mundhenke: Bei Mikrokalkassoziation des DCIS führen wir intraoperativ eine Präparatradiographie durch. Routinemäßig fertigen wir eine postoperative Mammographie an. Ist diese unauffällig, empfehlen wir mammographische Nachsorge- 
intervalle von zunächst 6 Monaten für die betroffene und jährliche Intervalle für die nicht betroffene Brust. Nach 2 Jahren verlängern wir die mammographischen Nachsorgeintervalle auf jährliche beidseitige Mammographien.

Marth: Die überwiegende Mehrzahl der Patientinnen mit DCIS wird aufgrund von Mikrokalzifikationen diagnostiziert. Damit spielt die Mammographie zweifellos die dominierende Rolle in der Diagnostik des DCIS. Die Kernspintomographie ist als zusätzliche Untersuchung für die Abschätzung der Ausdehnung sehr hilfreich. Jährliche Mammographieintervalle sind für die Nachsorge von Patientinnen mit DCIS ausreichend. Die Durchführung eines intraoperativen Präparatröntgens erlaubt den Nachweis der Entfernung aller Mikrokalzifikationen und stellt damit einen wichtigen Ausgangspunkt für die weiteren Nachsorgeuntersuchungen dar.

Tulusan: Die Mammographieintervalle müssen sich nach der Erfahrung des Auftretens der Lokalrezidive orientieren. Da rund $50 \%$ der Lokalrezidive in fast allen Studien invasive Karzinome sind, ist die engmaschige Kontrolle nach DCIS bei Patienten, die brusterhaltend behandelt wurden, unbedingt notwendig. So ist ein 6-monatiges Mammographieintervall in der Nachsorge für die ipsilaterale Seite obligat und ein jährliches Intervall für die kontralaterale Seite für die Zeit nach der DCIS-Diagnose.

Eigene Erfahrungen zeigen eine mediane rezidivfreie Zeit von 28 Monaten (6-60 Monate).

Jakisch: Wegen der berechtigten Sorge um das Auftreten von so genannten Intervallkarzinomen ist diese Frage in den letzten Jahren zu einem Dauerbrenner geworden. International gibt es hierzu leider keine einheitlichen Vorgehensweisen. Das gilt nicht nur für die Auswahl der geeigneten Altersgruppe, sondern auch für die Anzahl der durchzuführenden Mammographien und letztlich auch für die Intervalle. Das Mammographie-Screening in Deutschland sieht in der Altersgruppe ab dem 50. Lebensjahr ein 24-monatiges Intervall vor. Hier wird eine bilaterale Mammographie in zwei Ebenen durchgeführt. Für die intensivierte Krebsfrüherkennung der Brustdrüse beim familiären Mammakarzinom (BRCA-1+2-Mutation) wird ein 12-monatiges Intervall empfohlen.

\section{Frage 4: Welchen Stellenwert hat die Sofort- rekonstruktion bei ausgedehntem DCIS?}

Jonat/Mundhenke: Die überwiegende Zahl der Patientinnen mit einem DCIS wird bei uns brusterhaltend operiert, so dass häufig die Notwendigkeit der Rekonstruktion nicht gegeben ist. Wenn eine ablative Therapie notwendig wird, halten wir häufig eine sofortige Rekonstruktion für möglich. Bei besonderen Risikokonstellationen warten wir zunächst die endgültige histologische Befundung ab. Falls dann eine Radiatio notwendig erscheint und eine Rekonstruktion erwünscht ist, führen wir den Wiederaufbau erst nach der Bestrahlung durch.

Marth: Bei jenen Patientinnen, die ein ausgedehntes DCIS (VanNuys-Index 10 und mehr) aufweisen, stellt die Entfernung der Brust die Therapie der Wahl dar. Da sich DCIS-Areale häufig über größere Abschnitte der Brust ausdehnen, würde eine brusterhaltende Therapie auch keine guten kosmetischen Ergebnisse erwarten lassen. Aus diesem Grund stellt die hautschonende Mastektomie mit Wiederaufbau, z.B. mit Hilfe des freien Musculus gracilis-Lappens, eine Therapieoption dar, die exzellente onkologische und kosmetische Ergebnisse erzielt.

Jakisch: Eine Einschränkung zur Sofortrekonstruktion nach totaler Entfernung des Brustdrüsenkörpers besteht nur selten. Operateur und Patientin müssen sich nur darüber im Klaren sein, dass die Rate der Komplikationen, verglichen mit einem zweizeitigen Verfahren, erhöht sein kann. Dabei hat die Auswahl des Verfahrens in der Hand des Geübten keinen Einfluss auf die Komplikationsrate.

Bezogen auf das DCIS sehe ich eigentlich keine Kontraindikationen, da bei suffizienter präoperativer Diagnostik selten eine Radiatio in diesem Setting erforderlich ist. Aus meiner Sicht ist die Rekonstruktion mit Eigengewebe zu bevorzugen.

\section{Frage 5: Wann erfolgt beim DCIS eine Ablatio?}

Jonat/Mundhenke: Wir behandeln ein DCIS ablativ, wenn die Brust postoperativ bildgebend nicht überwachbar erscheint (z.B. bei ausgedehntem oder bei nicht mikrokalkassoziiertem DCIS), bei multifokalem DCIS oder bei einer ungünstigen Brust-Tumor-Relation. Auch bei Lokalrezidiven eines brusterhaltend vorbehandelten DCIS ziehen wir eine Ablatio der Brust häufiger in Betracht.

Stierer: Die Indikation zur Ablatio beim DCIS entspricht im Wesentlichen den Richtlinien beim invasiven Karzinom. Entscheidend ist dabei die Tumor-Brustgrößen-Relation. Bei der brusterhaltenden Operation sollte unter Erzielung eines ansprechenden kosmetischen Ergebnisses ein freier Schnittrand von 1-5 mm, im Idealfall von $10 \mathrm{~mm}$, erzielt werden. Diese Vorgangsweise kann bei Läsionen bis zu $3 \mathrm{~cm}$ (maximal $5 \mathrm{~cm}$ Durchmesser) gewählt werden. Ebenso ist bei disseminierten, pathologischen Mikrokalzifikationen, beim multizentrischen Befall (2 Herde in verschiedenen Quadranten der Brust) sowie bei Mitbefall der Mamille ein brusterhaltendes Verfahren nicht angezeigt.

Eine sekundäre Ablatio ist notwendig, wenn es nach einer sinnhaften Anzahl von Nachexzisionen nicht gelingt, tumorfreie Schnittränder zu erzielen. Bei bioptisch verifizierter Mikroinvasion und bei jedem ablativem Eingriff sollte eine 
Sentinellymphknotenbiopsie wegen der Möglichkeit bereits vorhandener lokaler Invasivität angeschlossen werden. Patientinnen mit ablativ behandelten DCIS stellen ideale Kandidatinnen für eine Sofortrekonstruktion der Brust dar.

Tulusan: Die Indikation für eine Ablatio ist sicherlich gegeben wenn trotz der entsprechenden operativen Therapieversuche keine tumorfreien Resektionsränder erreicht werden können oder wenn durch das Volumen der notwendigen Resektion keine kosmetisch akzeptable Brustform mehr erhalten werden kann. Trotz zusätzlicher Radiatio zeigt sich bei Non-insano-Resektionen eine sehr hohe Lokalrezidivrate (siehe Frage 1): Strahlentherapie kann eine unvollständige Chirurgie nicht ausgleichen! Für die Verwendung von onkoplastischen Rekonstruktionstechniken zum Volumenersatz nach großen Defekten der Brust zur Erreichung der tumorfreien Resektionsränder oder zur Wiederherstellung einer kosmetisch guten Brustform (z.B. Reduktionsplastiktechniken, Latissimus-dorsi-Muskel- oder Hautmuskel-Lappen usw.) statt einer Ablatio besteht keine Kontraindikation. Die eigene Erfahrung bei der Verwendung solcher Verfahren bei ausgedehnten DCIS-Fällen zeigt eine niedrige Lokalrezidivrate.

Jakisch: Dieses Verfahren gehört heute weiterhin zum therapeutischen Armamentarium in der primär operativen Therapie, aber genauso auch nach primär systemischer Chemotherapie. Früher ergab sich die Indikation zur Ablatio zwangsläufig aus den Kontraindikationen für eine Brusterhaltung. Heute sind es sicher noch die folgenden Punkte:

- Multizentrizität,

- R1-Resektion trotz wiederholter Nachresektion,

- inflammatorisches Mammakarzinom.

Durch die Einführung der «Skin-sparing»-Mastektomie haben sich die Indikationen weiter verschoben.

Bei der Durchführung der Mastektomie im klassischen Sinne sollte die Frage der sekundären Rekonstruktion in die Auswahl der Schnittführung mit einbezogen werden.

\section{Frage 6: Welchen Stellenwert hat der intraoperative Gefrierschnitt beim DCIS?}

Jonat/Mundhenke: Bei suspekten mammographischen Mikrokalkbefunden streben wir präoperativ durch Vakuumbiopsie eine histologische Abklärung an. Intraoperativ führen wir standardmäßig eine Präparatradiographie durch, verzichten jedoch bei DCIS-Befunden auf den intraoperativen Schnellschnitt. Anhand des endgültigen histologischen Befunds am Paraffinmaterial wird dann über eine weitergehende Therapie entschieden. Auch nach den Leitlinien der AGO (Arbeitsgemeinschaft Gynäkologische Gynäkologie / Mamma, 2006) erscheint die Durchführung einer intraoperativen Schnellschnittuntersuchung wenig sinnvoll.
Marth: Diese Frage ist mit einem einzigen Wort zu beantworten: Ablehnen.

Auf Grund der besonderen Gewebekonsistenz, Verkalkungen und hohem Fettanteil mit gleichzeitig nur minimalen Veränderungen in einzelnen Milchgängen lässt der Gefrierschnitt keine ausreichend sichere Beurteilung der Läsion zu. Aus diesem Grund muß zweifellos eine definitive Aufarbeitung nach Fixierung gefordert werden.

Stierer: Grundsätzlich ist die Durchführung eines Gefrierschnitts bei intraduktalem Karzinom nicht angezeigt. Bei einem DCIS in einer mastopathisch veränderten Brust, vor allem bei der prämenopausalen Frau, kann bei unklar verhärtetem Schnittrand zur Vermeidung einer unnötigen Nachexzision ein intraoperativer Gefrierschnitt in Absprache mit der Pathologie hilfreich sein.

Tulusan: Grundsätzlich bietet der intraoperative Gefrierschnitt («Schnellschnitt») die Möglichkeit, die Tumorresektion abzuklären und die freien oder nicht freien Resektionsränder morphologisch zu belegen. Das Ziel ist häufig eine «einzeitige Tumoroperation», um damit der Patientin mehrere Operationen zu ersparen. Selbst bei großer Erfahrung ist die Fehlerquote der Schnellschnittuntersuchung größer als bei Untersuchung im Paraffinschnittverfahren. Die wichtigste Voraussetzung ist, dass der Pathologe die entsprechende Erfahrung in der Schnellschnittdiagnostik der speziellen Organerkrankung hat und sich nicht scheut zu sagen: "Ich kann es nicht beantworten, Paraffinschnitte abwarten!». Wenn der Pathologe zusätzlich auch alle klinischen Befunde kennt, ist das sicherlich von Vorteil. Die präoperative interdisziplinäre Besprechung, wie beim zertifizierten Brustzentrum gefordert, ist hier notwendig. Unter den oben genannten Bedingungen und Prämissen kann der intraoperative Gefrierschnitt zur Überprüfung der lokalen Kurabilität sinnvoll verwendet werden. Wir haben dadurch auch beim DCIS in Kombination mit der intraoperativen Präparatradiographie die Häufigkeit der «zweiten» oder «dritten» Operation deutlich verringern zu können.

Jakisch: Durch die Einführung der minimal invasiven diagnostischen Verfahren und durch die Qualitätsmerkmale der Zertifizierungen erfolgt in unserer Klinik so gut wie keine intraoperative Dignitätsabklärung mittels Gefrierschnitt. Diese war und bleibt ausschließlich palpablen Läsionen vorbehalten und wurde und wird nicht zur Abklärung nonpalpabler Läsionen eingesetzt. Das gilt vor allem für den Einsatz der Gefrierschnittdiagnostik beim DCIS. Vorteilhaft ist sicher die direkte Umsetzung operativer Konsequenzen. Nachteilig bleiben die folgenden Punkte:

- geringere Sensitivität,

- Verlust von 25-50\% des Materials,

- Gefrierartefakte im Paraffinschnitt. 


\section{Teilnehmer}

Prof. Dr. med. Christian Jackisch

Klinik für Gynäkologie und Geburtshilfe

Klinikum Offenbach GmbH

Starkenburgring 66, 63069 Offenbach, Deutschland

Tel. +49 69 8405-3850, Fax -4456

E-mail Christian.Jackisch@klinikum-offenbach.de

Prof. Dr. med. Walter Jonat

PD Dr. med. Christoph Mundhenke

Klinik für Gynäkologie und Geburtshilfe

Universitätsklinikum Schleswig-Holstein, Campus Kiel

Michaelisstraße 16, 24105 Kiel, Deutschland

Tel. +49 431 59721-00, Fax -46

E-mail jonat@email.uni-kiel.de

E-mail cmundhenke@hotmail.com
Univ.-Prof. Dr. Christian Marth

Universitätsklinik für Frauenheilkunde

Tiroler Landeskrankenanstalten $\mathrm{GmbH}$

Anichstraße 35, 6020 Innsbruck, Österreich

Tel. +43 50 504230-51, Fax -55

E-mail christian.marth@uibk.ac.at

Univ. Prof. Dr. Michael Stierer

Abteilung für Chirurgie, Hanusch Krankenhaus

Heinrich-Collin-Straße 30, 1140 Wien, Österreich

Tel. +43 191021-2560, Fax -2654

E-mail michael.stierer@wgkk.sozvers.at

Prof. Dr. med. Augustinus Harjanto Tulusan

Frauenklinik

Klinikum Bayreuth

Preuschwitzerstr. 101, 95445 Bayreuth, Deutschland

Tel. +49 921400 5502, Fax -5509

E-mail tulusan.klinikum-bayreuth@t-online.de 